\title{
12 Lead Placement Non-Standard
}

National Cancer Institute

\section{Source}

National Cancer Institute. 12 Lead Placement Non-Standard. NCI Thesaurus. Code C71114.

An electrocardiogram (ECG) lead placement whereby the limb leads are placed on the torso for easier and faster application in emergency situations. 